\title{
ENERGY HARVESTING FOR EFFICIENT 5G NETWORKS
}

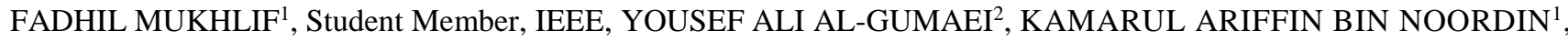 \\ ABDU SAIF AL-RASSAS ${ }^{1}$
}

${ }^{1}$ Department of Electrical Engineering, Faculty of Engineering, University of Malaya, Kuala Lumpur, Malaysia

${ }^{2}$ Faculty of Engineering, Al-Madinah International University, Selangor, Malaysia

\begin{abstract}
The design of traditional mobile wireless network emphasises on large capacity as well as ubiquitous access. However, environmental protection and energy saving may soon become inevitable trends due to rising global demands. In the wireless networking field, researchers should shift their focus towards efficient networks and energy harvesting (EH). In this work, we consider energy efficiency and $\mathrm{EH}$ to address the issue of energy shortage, which also represents the ratio of the quantity of data that have been transferred to the dissipated energy within the system, simultaneously. The simulation-based mathematics signifies the relation between energy efficiency $\left(C_{E}\right)$ against the training interval $\left(T_{t}\right)$ and how the energy efficiency is impacted by the change in block size and number of users.
\end{abstract}

Keywords-Resource Allocation, Energy Harvesting (EH), Information Decoding (ID), Green Transmission, Wireless Communication, Networking, $5 G$.

\section{INTRODUCTION}

It is expected that the next-generation wireless networks should offer access to high-speed Internet anytime and anywhere. The growing popularity of smartphones has hastened the process and has generated new traffic demands, such as gaming and mobile video. The need for ubiquitous access as well as rapidly growing data traffic has resulted in dramatic expansion of network infrastructures as well as rapid increase in energy demands. Hence, it is imperative for mobile operators to keep a sustainable capacity growth and simultaneously limit the electric bill [1] and [2].

For operating wireless communication systems, amassing energy from the environment and converting this energy to electrical power has now become an attractive possibility. Even though employing this method does not result in directly reducing the amount of energy needed to operate the system, it makes possible powering of wireless networks via clean energy and renewable sources [3]. In terms of wireless communications, there have been two key types of energy harvesting so far: Environmental and Radio Frequency [4], [5], [6] and [7]. Moreover, an intriguing possibility is offered by radio-frequency energy harvesting, which aids in decreasing the randomness related with the wireless power sources. The concept includes integrating both wireless power transfer and energy harvesting techniques. This enables energy sharing amongst network nodes [8], increasing the nodes' lifetime when battery energy is low [9] and [10]. The energy signals' superimposition on regular communication signals can aid in this approach, which results in the so-called 'power transfer and wireless information' [11] and [12]. In practical wireless communication systems, Channel State Information at the Transmitter (CSIT) can be obtained through the channel training process, but because of the estimation error, the obtained CSIT is not perfect. Such inadequacy of the process can be directly linked to deterioration of performance; however, its impacts on the EH model have not been investigated yet.

In this paper, the impact of the channel training process was investigated; in downlink networks, the error is determined for the energy efficiency where the receivers can perform EH. Hence, this study's objective is to provide an idea on EH technology and energy-efficient resource allocations, which promises to offer solutions to address the issues in providing ample energy so that wireless sensors can work continuously. We have made an attempt to establish synergistic effects and offer a solution to address the energy shortage crisis by integrating $\mathrm{EH}$ and allocation of energy-efficient resources.

The paper is structured as follows: Section 2 offers the definition on the system model. Section 3 offers a synopsis of energy harvesting. Section 4 offers a synopsis regarding simulation outcomes and related discussion. Section 5 provides the conclusion of the entire work and its objective.

\section{SYSTEM MODEL}

We assume that downlink networks include a single transmitter (Tx) and $K$ receivers ( $R x S)$, as shown in Fig. 1. A single antenna has been geared for each $T x$ and $R x s$. In each $R x$, the receiver Radio Frequency $(R F)$ signals can be employed for either Energy Harvesting $(\mathrm{EH})$ or Information Decoding (ID); however, employing $R F$ signals for $\mathrm{EH}$ and ID simultaneously is not possible [13]. In the received signal, an additive white Gaussian noise is associated with zero mean and variance $\sigma_{n}^{2}$, but the noise power is considered to be too small for harvesting energy. We have assumed a block-fading channel model with a constant channel for a particular 
transmission block $T$, but can change independently for each transmission block. $h_{k} \cong C N\left(0, \sigma_{n}^{2}\right)$ is used to represent the channel between $R x k$ and $T x$. At the start of each transmission block, signals from $R x S$ are trained by the $T x$ receivers to determine channel states for a training interval $T_{t}$. The $T x$ can be employed to determine the channels that have a minimum mean square error (MMSE) estimator according to the received training signals. The estimated channel can be represented as $h_{k}=h_{k}-e_{k}$, where the channel estimation error is signified by $e_{k}$. The error $e_{k}$ that includes the MMSE estimator is based on complex Gaussian distribution that has zero mean and variance $\sigma_{e}^{2}=\left(\sigma_{h}^{2} \sigma_{n}^{2}\right) /\left(\sigma_{n}^{2}+\sigma_{h}^{2} p_{t} T_{t}\right)$. Then, $h_{k} \cong C N\left(0, \sigma_{h}^{2}\right)$, where $\sigma_{h}^{2}=\sigma_{h}^{2}-\sigma_{e}^{2}$ is employed to evaluate according to the orthogonality principle for MMSE [14]. The following presents signal that is received by the user $K$ for both noise ratio and interference:

$$
\gamma_{k}=\frac{h_{k}^{2} p_{k}}{\sum_{j=1}^{k} h_{j}^{2} p_{j} \rho_{j, k}^{2} p_{j}+\sigma^{2}}
$$

The achievable sum-rate along with the estimated channel can be denoted by:

$$
\begin{aligned}
R\left(T_{t}, p_{k}\right) & =\sum_{k=1}^{K} r_{k} \\
& =\sum_{k=1}^{K} \frac{T-K T_{t}}{T} \log _{2}\left(1+\gamma_{k}\right) \\
& =\sum_{k=1}^{K} \frac{T-K T_{t}}{T} \log _{2}\left(1+\frac{h_{k}^{2} p_{k}}{\sum_{j=1}^{k} h_{j}^{2} p_{j} \rho_{j, k}^{2} p_{j}+\sigma^{2}}\right)
\end{aligned}
$$

where $p_{k}$ represents the transmission power regarding data transmission. Moreover, $\sigma^{2}=\sigma_{n}^{2} / \sigma_{h}^{2}$ has been defined for variance and in (2), $T-K T_{t} / T$ signifies the loss of time in data transmission due to time $T_{t}$ committed for training the channel. Since self-interference due to channel estimation error can be considered as noise, the effective noise power can be considered similar to the sum of the self-interference power $p_{d} \sigma_{e}^{2}$ as well as the additive noise power $\sigma_{n}$ [15]. Accordingly, as there is growth in $T_{t}$, there is an increase in the effective signal to noise ratio (SNR) when there is data transmission occurring, but there is also reduction in the time for data transmission. Based on this trade-off relationship, for maximising $r_{k}$, an optimal value of $T_{t}$ is present.

According to the estimated channel $h_{k}$, the harvested energy for $R x s$ that can be expected is:

$$
G\left(T_{t}, p_{k}\right)=\sum_{k=1}^{K} \frac{T-k T_{t}}{T} \eta_{k}\left|h_{k}\right|^{2} p_{k}
$$

where $\eta_{k}$ represents the achieved energy conversion efficiency due to conversion of the received $R F$ signal into harvested energy with the help of $R x k$. The inequality $\sum_{k=1}^{K} \eta_{k}\left|h_{k}\right|^{2} \leq 1$ should hold, since the amount for the total harvested energy should not be more than the transmitted energy. The net energy dissipation in the system is decreased due to the harvested energy $G\left(T_{t}, p_{k}\right)$ as shown below:

$$
\begin{aligned}
C\left(T_{t}, p_{k}\right) & =p_{s}+\frac{k T_{t}}{T} p_{t}+\frac{T-k T_{t}}{T} p_{k}-G\left(T_{t}, p_{k}\right) \\
& =p_{s}+\frac{k T_{t}}{T} p_{t}+\frac{T-k T_{t}}{T} p_{k}-\sum_{k=1}^{K} \frac{T-k T_{t}}{T}\left|h_{k}\right|^{2} p_{k}
\end{aligned}
$$

where $p_{s}$ represents continuous energy consumption in circuits, $p_{t}$ signifies channel training's transmission power and $\left(T-k T_{t} / T\right) p_{k}$ and $\left(k T_{t} / T\right) p_{t}$ denote the consumption of power for data transmission and determining channels, respectively. As a performance metric, $C_{E}=$ $R\left(T_{t}, p_{k}\right) / C\left(T_{t}, p_{k}\right)$ denotes the entire system's energy efficiency [13]. The energy efficiency $C_{E}$ suggests the rate per unit energy (bits/s/J) that can be achieved, i.e. it demonstrates the utilisation of efficiency energy during data transmission.

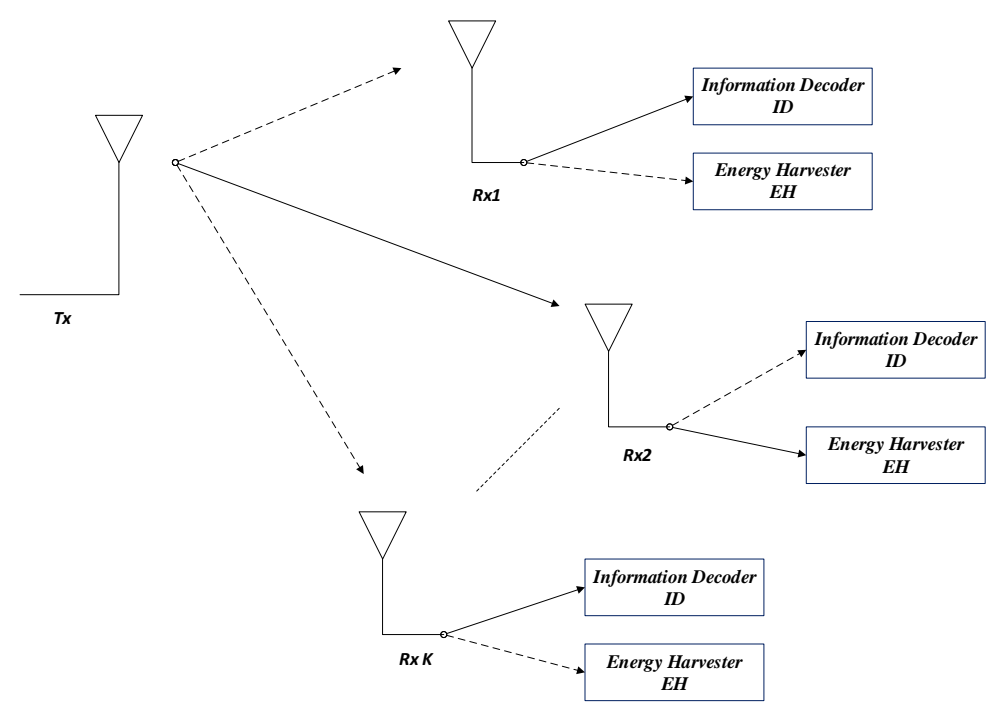

Fig. 1. System Model. 


\section{ENERGYS EFFICIENT NETWORKS}

According to various studies, harvesting of both wind and solar energy can be employed to operate communication systems [4] and [5]. The focus here has to be on how direct improvement in the efficiency of system energy is not achieved by this technique, since there is no decrease in the quantity of the much-needed energy for functionalising the system. However, this is still considered an enticing option since powering wireless networks is allowed with the help of clean energy and renewable sources, thereby providing a virtually unlimited energy supply, while minimising $\mathrm{CO}_{2}$ emissions. However, radio signals over the air can also be employed to harvest energy [6]. This lets the network nodes to employ various techniques for wireless power transfer for energy sharing amongst each other, with an aim to wirelessly recharge nodes with low battery power [9], [12], [16] and [15]. However, the main aim of the current and future cellular networks would be to provide high safety, higher data rates and universal communication that vouch for quality of service (QoS).

However, maximising the energy efficiency represents a resource allocation problem. The following optimisation issue can be solved to get optimal resource allocation strategies by:

$$
\begin{gathered}
\max _{T_{t}, p_{k}} \frac{R\left(T_{t}, p_{k}\right)}{C\left(T_{t}, p_{k}\right)} \\
\text { s.t. } C 1: r_{k} \geq r_{\text {min }} \quad \forall k \\
\text { C2: } g_{k} \geq g_{\min } \quad \forall k \\
\text { C3: } p_{k} \leq p_{\max } \\
\text { C4: } p_{k} \geq 0 \\
\text { C5: } 0 \leq T_{t} \leq T
\end{gathered}
$$

Nevertheless, sending information and energy simultaneously cannot be achieved in practical application, since the information gets destroyed due to energy harvesting operation done in the radio frequency domain. To realise SWIPT, a method is available, in which splitting of the received signal should be done separately in two different parts. The first part includes harvesting of the energy, while the other part includes decoding of information. Higher energy efficiency can be achieved through the SWIPT method since it can employ the harvested power again to continue with further transmission. This allows optimising the energy efficiency (EE) issue associated with the power transfer (SWIPTMultiple Input Multiple Output (MIMO)) systems and, at the same time, providing wireless information that also has statistical channel state information (CSI) feedback. Should there be a need for further transmission for the energy harvesting node, within the system capacity, measurement is done for the potential capacity created due to transferred energy [17]. Besides the fact that harvesting results have suggested the superiority of the proposed schemes on EE, the EE system can still be improved further [17]. In [18], the SWIPT system as well as its use in wireless cooperative networks has been investigated, where each signals sent from the source were employed to power up the energy harvesting (EH) relay. However, it should be noted that the application of SWIPT should be considered for the cooperative clustered wireless sensor networks (WSNs) [19] and [20]. In [21], for wirelessly delivering the renewable green energy and safeguarding the information in mobile receivers for the distributed antenna communication systems, formulation of the resource allocation algorithm was done. Moreover, for heterogeneous wireless networks, wireless information and power transfer (SWIPT) was established simultaneously [22] and [23].

\section{SIMULATION RESULTS AND DISCUSSION}

For the purpose of simulations, an indoor communication system with $K=6$ receivers are considered. Table 1 provides a list of detailed simulation environment. The results present the energy efficiency $C_{E}$, which is against the training interval $T_{t}$ relating to the Block Size $T$ shown in Fig. 2 as well as to the number of receivers $K$ presented in Fig. 3.

However, such dependence of $T_{t}$ on $T$ should also be noted and $K$ is on contrast when compared with the well-known result for the optimal training when considering a simple point-to-point network. Here, the optimal training duration to maximise data rate can be considered similar to the total number of transmit antennas, irrespective of $T$ and SNR. Further, the $C_{E}$ is turning out to be low for large $T_{t}$ as presented in Fig. 2. This is due to the amount of energy dissipation, which results in deteriorating the most significant factor $C_{E}$.

On the other hand, in Fig. 3, it can be seen that a much larger quantity of energy can be harvested with $K=6$ when compared with $K=1$. This is because in this case, $K$ is larger than 1 because of one of the users decoding the information while the other users harvest energy. However, for a single user case involving $K=1$, for information transmission, the transmission time taken by the user to harvest energy is not used.

TABLE I. SIMULATION ENVIRONMENTS

\begin{tabular}{|c|c|}
\hline Parameters & Value \\
\hline Target system & Indoor Communication \\
\hline Circuit energy consumption & $p_{s}=5 \mathrm{dBm}$ \\
\hline Training signal power & $p_{t}=23 \mathrm{dBm}$ \\
\hline Energy conversion efficiency & $\eta_{k}=0.9$ \\
\hline Path loss & $h_{k}=1$ \\
\hline cross correlation coefficients & $\rho_{j, k}^{2}=0.01$ \\
\hline
\end{tabular}




\begin{tabular}{|c|c|}
\hline Number of receiver & $K=6$ \\
\hline Transmit power & $p_{k}=10 \mathrm{dBm}$ \\
\hline Background noise & $\sigma^{2}=1$ \\
\hline Block Size & $T=5000$ \\
\hline Required data rate & $r_{\min }=10 \mathrm{bps} / \mathrm{Hz}$ \\
\hline Required harvested energy & $g_{\min }=-20 \mathrm{dBm}$ \\
\hline
\end{tabular}

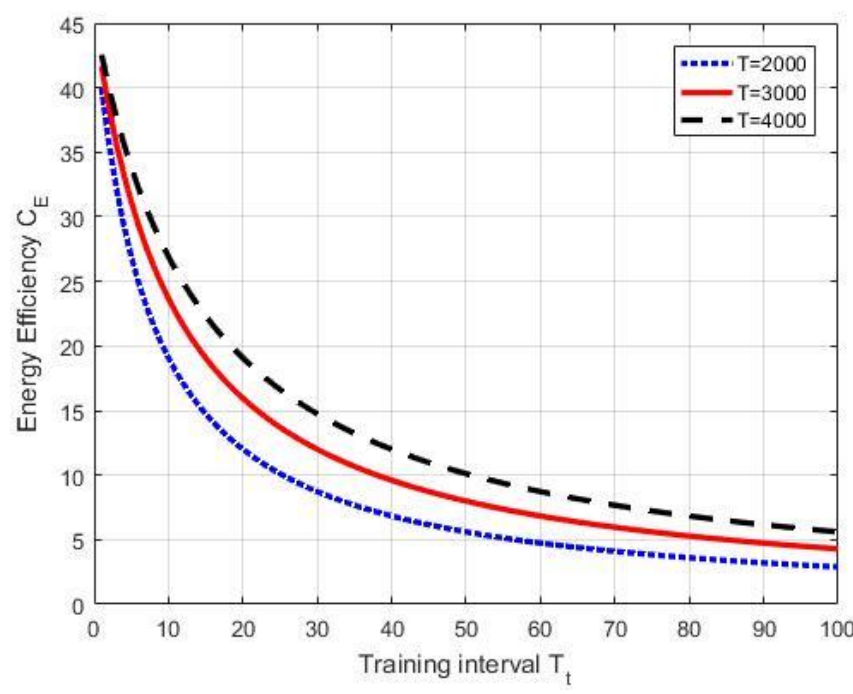

Fig. 2. $\mathrm{C}_{\mathrm{E}}$ versus $\mathrm{T}_{\mathrm{t}}$ with Respect to Block Size $\mathrm{T}$.

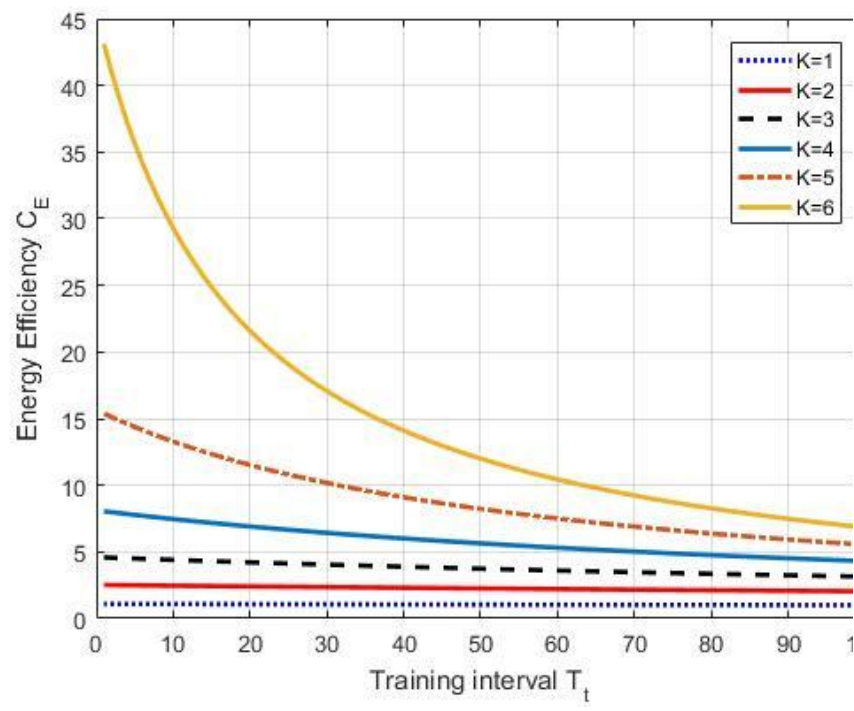

Fig. 3. $C_{E}$ versus $T_{t}$ with Respect to The No. of Receivers $K$.

\section{CONCLUSION}

In this paper, the efficiency of harvesting energy through Radio Frequency (RF) signals is examined in wireless

$$
\text { (RF) signals is examined in wireless }
$$

networks by employing a mathematical model built on realistic assumption based on imperfect CSIT. According to the mathematical model, a less computational complexity formula is being used to investigate energy efficiency prevalent in an indoor wireless network. Although harvesting ample energy from radio signals for operating wireless networks has not been made possible yet due to numerous hardware limitations, the results encourage addressing this myth in the near future. This research study's future direction would be on focusing to develop new mathematical algorithm and alter it in a bid to achieve green radio environment.

\section{ACKNOWLEDGEMENTS}

This work is supported by university of Malaya, Faculty of Electrical Engineering.

\section{REFERENCES}

A. De Domenico, E. Calvanese Strinati, and A. Capone, "Enabling Green cellular networks: A survey and outlook," Computer Communications, vol. 37, pp. 5-24, 1/1/ 2014.

Y. Chen, S. Q. Zhang, S. G. Xu, and G. Y. Li, "Fundamental Trade-offs on Green Wireless Networks," Ieee Communications

R. Mumford, "5G Manifesto for Deployment of 5G in Europe," ed: HORIZON HOUSE PUBLICATIONS INC 685 CANTON ST, NORWOOD, MA 02062 USA, 2016.

H. A. H. Hassan, L. Nuaymi, and A. Pelov, "Renewable energy in cellular networks: A survey," in 2013 IEEE Online Conference on Green Communications (OnlineGreenComm), 2013, pp. 1-7.

S. Ulukus, A. Yener, E. Erkip, O. Simeone, M. Zorzi, P. Grover, et al., "Energy Harvesting Wireless Communications: A Review of Recent Advances," IEEE Journal on Selected Areas in Communications, vol. 33, pp. 360-381, 2015.

X. Lu, P. Wang, D. Niyato, D. I. Kim, and Z. Han, "Wireless Networks With RF Energy Harvesting: A Contemporary Survey," IEEE Communications Surveys \& Tutorials, vol. 17, pp. 757-789, 2015.

H. J. Visser and R. J. M. Vullers, "RF Energy Harvesting and Transport for Wireless Sensor Network Applications: Principles and Requirements," Proceedings of the IEEE, vol. 101, pp. 14101423, 2013.

L. Liu, R. Zhang, and K.-C. Chua, "Wireless information transfer with opportunistic energy harvesting," IEEE Transactions on Wireless Communications, vol. 12, pp. 288-300, 2013.

B. Gurakan, O. Ozel, J. Yang, and S. Ulukus, "Energy Cooperation in Energy Harvesting Communications," Ieee Transactions on Communications, vol. 61, pp. 4884-4898, Dec 2013.

Y. K. Chia, S. M. Sun, and R. Zhang, "Energy Cooperation in Cellular Networks with Renewable Powered Base Stations," Ieee Transactions on Wireless Communications, vol. 13, pp. 69967010, Dec 2014.

K. B. Huang and E. Larsson, "Simultaneous Information and Power Transfer for Broadband Wireless Systems," Ieee Transactions on Signal Processing, vol. 61, pp. 5972-5986, Dec 2013.

D. W. K. Ng, E. S. Lo, and R. Schober, "Wireless Information and Systems," Ieee Transactions on Wireless Communications, vol. 12, pp. 6352-6370, Dec 2013.

K. Lee and J. P. Hong, "Energy-Efficient Resource Allocation for Simultaneous Information and Energy Transfer With Imperfect Channel Estimation," IEEE Transactions on Vehicular Technology, vol. 65, pp. 2775-2780, 2016. Magazine, vol. 49, pp. 30-37, Jun 2011. Power Transfer: Energy Efficiency Optimization in OFDMA 
[14] B. Hassibi and B. M. Hochwald, "How much training is needed in multiple-antenna wireless links?," IEEE Transactions on Information Theory, vol. 49, pp. 951-963, 2003.

[15] Y. A. Al-Gumaei and K. Dimyati, "Optimal power control game for primary-secondary user in cognitive radio network," International Journal of Physical Sciences, vol. 5, pp. 345-351, Apr 2010.

[16] A. Zappone and E. A. Jorswieck, "Energy-efficient resource allocation in future wireless networks by sequential fractional programming," Digital Signal Processing, vol. 60, pp. 324-337, $1 / / 2017$.

[17] Q. Sun, L. Li, and J. Mao, "Simultaneous Information and Power Transfer Scheme for Energy Efficient MIMO Systems," IEEE Communications Letters, vol. 18, pp. 600-603, 2014.

[18] Z. Chu, M. Johnston, and S. Le Goff, "SWIPT for wireless cooperative networks," Electronics Letters, vol. 51, pp. 536-538, 2015.

[19] S. Guo, F. Wang, Y. Yang, and B. Xiao, "Energy-Efficient Cooperative Tfor Simultaneous Wireless Information and Power Transfer in Clustered Wireless Sensor Networks," IEEE Transactions on Communications, vol. 63, pp. 4405-4417, 2015.

[20] W. Yang, W. Mou, X. Xu, W. Yang, and Y. Cai, "Energy efficiency analysis and enhancement for secure transmission in SWIPT systems exploiting full duplex techniques," IET Communications, vol. 10, pp. 1712-1720, 2016.

[21] D. W. K. Ng and R. Schober, "Secure and Green SWIPT in Distributed Antenna Networks With Limited Backhaul Capacity," IEEE Transactions on Wireless Communications, vol. 14, pp. 5082-5097, 2015.

[22] S. Akbar, Y. Deng, A. Nallanathan, M. Elkashlan, and A. H. Aghvami, "Simulatneous Wireless Information and Power Transfer in K-tier Heterogeneous Cellular Networks," IEEE Transactions on Wireless Communications, vol. 15, pp. 5804$5818,2016$.

[23] Y. Dong, M. J. Hossain, and J. Cheng, "Joint power control and time switching for SWIPT systems with heterogeneous QoS requirements," IEEE Communications Letters, vol. 20, pp. 328331, 2016. 